\title{
Die Prozesslandkarte zeigt den Weg zur lernenden Organisation
}

\author{
Paul Brandl, Christine A. Heyduk, Judith Stumpf
}

Ein Lernprojekt an der Fachhochschule Oberösterreich offenbarte, welche Produktivitätssteigerungen in Pflegeheimen möglich sind, wenn Geschäftsprozesse optimiert werden. Eine gute Grundlage dazu bietet das Erstellen einer Prozesslandkarte, die alle Beteiligten und Abläufe visuell darstellt.

Die Studierenden des Studienganges Sozialmanagement an der Fachhochschule Oberösterreich in Linz wenden im vierten Semester ihr erworbenes theoretisches Wissen im Bereich der Organisationslehre in der Praxis an.

Im letzten Sommersemester hatten wir mit den Führungskräften der Seniorenzentren Linz GmbH vereinbart, dass sie ihre Organisation als Lernpartner zur Verfügung stellen. Ausgangspunkt der gemeinsamen Überlegungen für das Lernprojekt war die nachfolgend dargestellte Prozesslandkarte (1) eines Alten- und Pflegeheimes (vgl. Abb.). Die Prozesslandkarte bildet die Leistungsprozesse eines Altenheimes ab - im Sinne der Prozessorganisation unterteilt nach Kern-, Support- und Steuerungsprozessen.

Gemeinsam mit den Geschäftsführern und der Pflegedirektorin der Seniorenzentren Linz GmbH wählten wir als Lehrveranstaltungsleiter insgesamt sechs Prozesse (je einer pro Studierendengruppe) aus, in denen nach Ansicht dieser drei Führungskräfte Optimierungspotenzial besteht:

- Beschaffung von Büromaterial und Pflegeprodukten

- Haustechnik

- Medikamentenversorgung

- Personalaufnahme

- Tageszentrum

- Wäscheversorgung
Der generelle Arbeitsauftrag seitens der Fachhochschule an die Studentinnen und Studenten lautete:

- Stellen Sie den Ist-Ablauf visuell dar und

- sammeln Sie die entlang dieses Prozesses von den handelnden Personen genannten Probleme.

- Anschließend sammeln Sie Ideen für den Soll-Zustand; teils anhand von Lösungen in anderen Organisationen (Best practice), teils durch Ideensammlung der Mitarbeiterinnen und Mitarbeiter der Seniorenzentren Linz GmbH. Entwickeln Sie daraus eigene Optimierungsvorschläge.

- Erstellen eines Berichts und einer Präsentation für die Auftraggeber

Ziel des Studienprojektes war es jedoch nicht, die erarbeiteten Vorschläge auch selbst zu realisieren.

Begleitend hatten die Studierenden im Rahmen der Lehrveranstaltung die Möglichkeit, theoretische Inputs zum Prozessmanagement, insbesondere zum Erkennen von kritischen Abläufen, zum visuellen Darstellen von Istund Soll-Prozessen sowie auch Beratungsleistung hinsichtlich der methodischen Vorgangsweise abzurufen. Die Aktivitäten sollten unter Anwendung der Instrumente des Projektmanagements (Projektauftrag, Projektstrukturplan, Statusberichte etc.) erfolgen.

Mittels Beobachtung, Interviews, Begehungen und der Sichtung von Formularen wurden zunächst Daten gesammelt. Damit konnten die Studierenden den Arbeitsablauf (= IstSituation) visuell gut darstellen, die Probleme zuordnen, eine Diagnose vornehmen und eigene Ideen entwickeln. Die Arbeitsteilung innerhalb der Gruppe erfolgte durch Selbstorganisation.

Die Darstellung der Ist-Prozesse erfolgte mit den Computer-Program-

\author{
Mag. Dr. Paul Brandl lehrt als \\ Professor an der Fachhoch- \\ schule Oberösterreich in Linz \\ Betriebswirtschaftslehre mit \\ Schwerpunkt Personal und Or- \\ ganisation. Zusammen mit \\ seinen beiden Kolleginnen \\ Christine A. Heyduk und \\ Judith Stumpf führte er mit \\ seinen Studierenden die hier \\ beschriebene Organisationsbe- \\ ratung der Seniorenzentren \\ Linz GmbH durch. \\ E-Mail Paul.Brandl@fh-linz.at
}

men Powerpoint, Excel oder Visio. Dies diente dem Zuordnen der Probleme und Identifizieren von Konfliktzonen einzelner Arbeitsschritte. Üblicherweise ist bei dieser lösungsorientierten Vorgangsweise erst der Soll-Ablauf genauer darzustellen, um dann Antworten auf die folgenden Fragen $\mathrm{zu}$ finden:

- Wie sieht der neu konzipierte Ablauf aus?

- Welche neuen Abläufe und Zuständigkeiten ergeben sich daraus?

- Welche Ressourcen sind notwendig?

- Welche Formulare werden benötigt?

- Welche Informationen werden wie an wen weitergeleitet?

In den Arbeitsgruppen wurden eine Reihe von Erkenntnissen und Ergebnissen erarbeitet. Nachfolgend ein Auszug: 


\section{Optimierungsvorschlag: Beschaffung von Büromaterial und Pflegeprodukten}

Die Studierenden erhoben in zwei Seniorenzentren die Wege der Bestellungen, des Materials und der Rechnungen. Sie dokumentierten die Schwierigkeiten des Pflegepersonals, aber auch die der zuliefernden und administrativ befassten Stellen. Hier ging der Verbesserungsvorschlag in Richtung einer dezentralen Auslieferung - nach Bedarf und unter Ausnutzung von Wertgrenzen:

- Jedes Stockwerk bestellt direkt beim Lieferanten im Rahmen des vorgegebenen Budgets mit eigenem Formular, damit die Lieferung eindeutig identifiziert und zugeordnet werden kann.

- Die Verwaltung erhält nur eine Monatsrechnung von einem Lieferanten.

- Die Lieferung erfolgt direkt in die Abteilungen, sodass sich das Abholen im Lager oder im Erdgeschoss erübrigt.

- Das Ausborgen von fehlendem Material aus anderen Abteilungen entfällt ersatzlos und ist auf den Ausnahmefall beschränkt.

\section{Optimierungsvorschlag: Haustechnik}

Die mit der Haustechnik befasste Gruppe von Studentinnen und Studenten untersuchte zwei Häuser. Aus Sicht der Arbeitsgruppe waren in einem Haus die Arbeitsabläufe bei Reparaturen sehr gut organisiert, während diese Abläufe im zweiten Haus bis zur Erledigung lange dauerten. Eine Verbesserungsmöglichkeit für alle Seniorenzentren sah die Gruppe bei der Meldung und Abarbeitung von Reparaturaufträgen via E-Mail an die Haustechnik. Langfristig wäre die Einführung einer Instandhaltungssoftware überlegenswert. Für die Beschaffung teurer Ersatzteile wurde eine Vereinfachung und damit Beschleunigung empfohlen und zwar mittels geänderter Kompetenzregelungen.

\section{Optimierungsvorschlag: Medikamentenversorgung}

Im Sinne einer Nahtstellenbetrachtung wurde eine ortsansässige Apo- thekerin bei der Erhebung des derzeitigen Ablaufes (von der Abteilung über die Ordination zur Apotheke) im Standardfall und im Akutfall miteinbezogen. Nachdem ein »Medienbruch « bei der Datenermittlung festgestellt wurde, ersetzt nun eine per E-Mail übermittelte Excel-Tabelle die bisher via Papier und Fax vorgenommenen Bestellungen. Eine Informationsschleife für die jeweilige Abteilung zur Erhöhung der Handlungssicherheit wurde zusätzlich eingebaut. Der Vorschlag der Studierenden bringt nach ihren Erhebungen und Berechnungen eine Zeitersparnis von zwei Stunden pro Woche und Abteilung.

\section{Optimierungsvorschlag: Personalaufnahme}

Da die Seniorenzentren Linz mit Jahresbeginn 2006 ein eigenständiges Unternehmen geworden sind, hat sich auch die Möglichkeit geboten, den Prozess der Personalaufnahme zu analysieren. Die Studierenden haben im Zuge der Projektarbeit erhoben, dass ein Software-Paket eingeführt werden soll. Sie beschränkten sich deshalb auf das Erstellen von Anforderungen an das Customizing im Zuge der Einführung der Software. Auch stellten sie die Frage, von welchem Personenkreis welche Personalinformationen noch als physische Personalakte benötigt werden. Vorschlag: eine dezentrale Dokumentation, die zentral abrufbar ist.

\section{Optimierungsvorschlag: Tageszentrum}

Schwerpunkt der Studierenden war hier die Frage, wie die Kommunikation beim Abholen von Tagesgästen von Zuhause einfacher gestaltet werden könnte. Für die Fahrer entstehen beim Abholen der Besucher von Tageszentren des Öfteren längere Wartezeiten, wenn beispielsweise Klienten nicht abgemeldet sind und die Türe nicht öffnen. Hier wurden Vorschläge zur pünktlichen Abholung, einer klientenadäquaten und trotzdem ressourcenschonenden Vorgangsweise - und letztlich auch für eine bessere Auslastung des Tageszentrums - erarbeitet.

\section{Optimierungsvorschlag: Wäscheversorgung}

Wäschestücke der Bewohnerinnen und Bewohner können im Zuge der Reinigung verloren gehen, werden falsch einsortiert oder an eine falsche Adresse zurückgeliefert. Verärgerung bei den Bewohnerinnen und Bewohner, den Angehörigen und dem Personal ist die Folge. Dies kostet Arbeitszeit und damit schwer quantifizierbare Kosten. Ein weiterer gravierender Aspekt: Es gibt fünf Lieferanten, die die sortierte Wäsche zu unterschiedlichen Zeitpunkten der Woche in fünf unterschiedlichen Behältnissen abholen. Fehlsortierungen haben höhere Kosten zur Folge, da für die Reinigung dieser Stücke höhere Tarife verrechnet werden. Die Instruktion der Mitarbeiterinnen und Mitarbeiter hinsichtlich der Sortierung ist daher besonders wichtig. Die Lösung lag darin, die Zahl der Lieferanten zu reduzieren, um Fehlsortierungen zu vermeiden. Die Sortierung nach Wäscheart erfolgt erst durch die Wäscherei und diese liefert die gereinigten Textilien an den vereinbarten Übergabepunkt in der Abteilung. Schriftliche Standardbestellungen sollten entfallen und durch stichprobenartige Kontrollen verbunden mit Vertragsstrafen für den Lieferanten ersetzt werden. Zusätzliche Bestellungen bedürfen weiter der "Schriftform « per E-Mail. Die Studierenden lehnten sich dabei an eine in einem Krankenhaus der Umgebung praktizierte und gut funktionierende Lösung an.

\section{Alle haben ihre Lektionen gelernt}

Die Studentinnen und Studenten haben in ihrer Rolle als externe Berater gelernt, dass

- Unzulänglichkeiten in der Arbeitsweise von außen nur dann wahrgenommen werden, wenn sie im Ergebnis wirksam oder kommuniziert sind

- das Einhalten von Vereinbarungen eine wesentliche Voraussetzung für gelingende Teamarbeit ist und Instrumente des Projektmanagements unterstützend wirken

- eine genaue Auftragsklärung sowie die Formulierung der Ziele und Nichtziele eine wesentliche Voraussetzung für gute Ergebnisse ist 


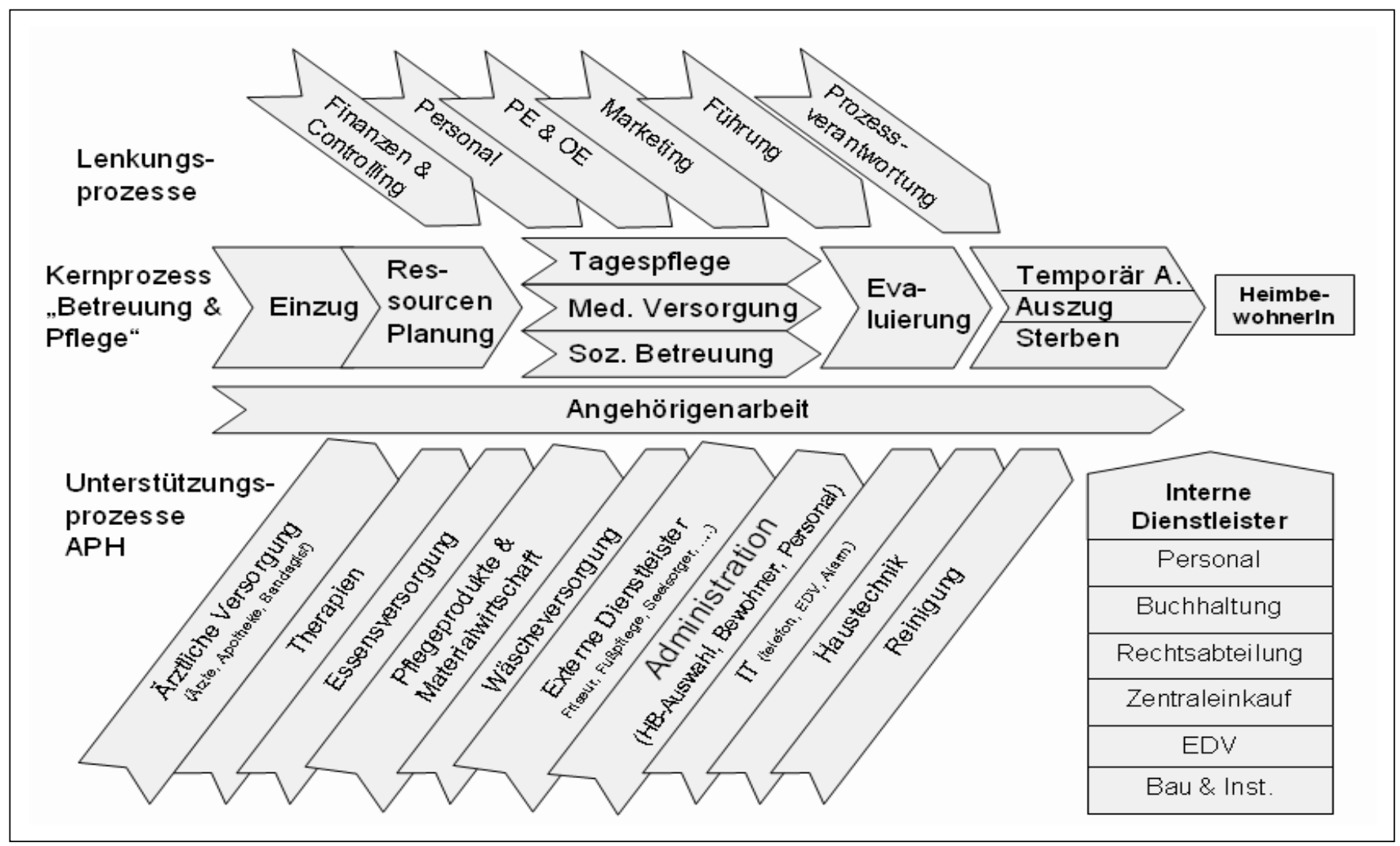

Mit einer Prozesslandkarte einer Organisation, beispielsweise einem Pflegeheim, können die Geschäftsprozesse, Ziele, Eingaben und Ergebnisse dieser Einrichtung offen gelegt werden.

- das Erkennen von Problemen nicht automatisch zu deren Behebung führt

Die Führungskräfte und die Mitarbeiterschaft der Seniorenzentren haben

- die Prozesslandkarte als einfache Darstellungsform der Leistungsprozesse kennengelernt

- anhand der Prozesslandkarte gemeinsam jene Prozesse ausgewählt, in denen Verbesserungspotenzial vermuteten

- durch die Betrachtung der Organisation in Ebenen erfahren, dass es bei Verbesserungen nicht um die Suche nach Schuldigen geht, sondern die sachliche Diskussion im Vordergrund steht

- erkennen können, dass es viele kleine Verbesserungen benötigt, um die Mitarbeiterinnen und Mitarbeiter weiter zu qualifizieren und die Organisation weiter zu entwickeln

- durch die Außensicht eine Reihe von Anregungen erhalten, die in weiteren Arbeitsgruppen noch umgesetzt werden können

Wir als Leitende der Lehrveranstaltung haben erfahren, dass

- die Betrachtung eines Unternehmens in drei Ebenen - Organisation, Prozess, Aufgabe - für die personenunabhängige Betrachtung hilfreich ist

- im Sinne eines lösungsorientierten Vorgehens unter Anwendung des Modells der Prozessorganisation alte Probleme durch die neuen Abläufe per se eliminiert werden

- die gefundenen Lösungen kurz-, mittel- und langfristig gedacht werden müssen

- es eines Prozessverantwortlichen bedarf, der die Weiterentwicklung steuert, den Prozess jährlich auditiert und zusammen mit den Mitarbeitenden optimiert.

\section{Resümee}

Die Prozesslandkarte als visuelle Darstellung der Leistungsprozesse und zentrales Instrument der Verständigung ermöglicht den Führungs- kräften sozialer Unternehmen eine ständige Verbesserung von Organisation, Prozessen und Aufgabenerledigungen. Voraussetzung dazu ist nicht nur eine »Es-wird-schon- gehen-

Haltung « der Führungskräfte bis zur Umsetzung, sondern auch eine entsprechende Qualifizierung der Mitarbeiterinnen und Mitarbeiter und gegebenenfalls ein (interner) Berater als Lernbegleiter. Die sachliche und pragmatische Vorgangsweise ermöglicht es außerdem, ohne Schuldzuweisungen für einen unbefriedigenden Ist-Zustand einen gemeinsam entwickelten Soll-Zustand anzustreben, vielleicht auch in mehreren Schritten. Die Folge: Mehr Produktivität, (viel) weniger Frustration, mehr Zeit für die Kunden und Nutzer.

\section{Anmerkungen}

(1) Vgl. Schmelzer, H. J./Sesselmann, W., Geschäftsprozessmanagement in der Praxis, 4. Auflage, 2004, Seite $55 \mathrm{ff}$.

(2) Vgl. Brandl, P., Produktivitätsentwicklung. Analyse-Instrumente praktisch, einfach und wirksam(2), Newsletter Sozialmanagement, 1/2006. 